\title{
Computational and Experimental Investigation of Supersonic Convection over a Laser-Heated Target
}

\author{
Eric C. Marineau* and Joseph A. Schetz \\ Virginia Polytechnic Institute and State University, Blacksburg, Virginia 24061 \\ and \\ Reece E. Neelı \\ AeroSoft, Inc., Blacksburg, Virginia 24061 \\ DOI: $\underline{10.2514 / 1.33697}$
}

\begin{abstract}
The cooling effect of a turbulent supersonic boundary layer over a laser-heated flat plate was investigated experimentally and numerically. Experiments were in the Virginia Polytechnic Institute and State University unheated supersonic wind tunnel at Mach 4. An absorbed laser power between 65 and $120 \mathrm{~W}$ was used, leading to a maximum heat flux between 10 and $19 \mathrm{MW} / \mathrm{m}^{2}$ at the center of the 4-mm-diam Gaussian beam. The surface and backside temperature distributions were measured using a midwave infrared camera and type-K thermocouples. The GASP conjugate heat transfer algorithm coupling the Navier-Stokes and the solid conduction equations was used to simulate the experiments. The main experimental results were as follows: Asymmetry in the surface temperature increases with laser power. Maximum cooling near the beam center varies linearly with laser power, in which the proportionality constant corresponds to the ratio of convective cooling to laser heating. For both the 65 and $81 \mathrm{~W}$ cases, cooling is somewhat underpredicted at the surface near the center, but agreement improves with distance and on the backside.
\end{abstract}

\section{Introduction}

D IRECTED-ENERGY weapons have been of interest ever since antiquity. Archimedes is said to have repelled a Roman attack by focusing sunlight using an array of parabolic mirrors, causing the approaching ships to catch fire. In recent decades, remarkable progress has been made since the first invention of the solid-state laser in the 1960s through gas dynamic, chemical, free-electron, and advanced solid-state lasers pushing today's state of the art [1] such that the use of directed energy as an effective weapon is a reality that is now being implemented through programs such as the Airborne Laser,, a system used to destroy ballistic missiles in their boost phase close to their launch area. The temperature increase of the target due to the laser heating is expected to be affected by the supersonic flow over it. To our knowledge, no experimental study of a laser-heated target subjected to a supersonic flow has ever been performed. The first objective of this research is to design and perform an experiment to measure the effect of turbulent supersonic flow on the temperature distribution on the immersed and back surfaces of a laser-heated target. Second, the experimental results are used for code validation as conjugate heat transfer (CHT) problem simulations are performed and compared with the experimental results.

Part of this investigation requires modeling the effect of a sharp variation in wall temperature on a supersonic boundary layer. Experimental data for a supersonic boundary layer subjected to a sharp variation in wall temperature are uncommon. Debieve et al. [2] performed measurements of the temperature and velocity profiles for a turbulent supersonic boundary layer with a step in wall to recovery temperature ratio $T_{w} / T_{r}$ of 1.5 and 2 . Using the measured velocity

Presented as Paper 4147 at the 39th AIAA Thermophysics Conference, Miami, FL, 25-28 July 2007; received 25 July 2007; revision received 19 February 2009; accepted for publication 20 February 2009. Copyright (C) 2009 by the American Institute of Aeronautics and Astronautics, Inc. All rights reserved. Copies of this paper may be made for personal or internal use, on condition that the copier pay the $\$ 10.00$ per-copy fee to the Copyright Clearance Center, Inc., 222 Rosewood Drive, Danvers, MA 01923; include the code 0887-8722/09 \$10.00 in correspondence with the CCC.

${ }^{*}$ Currently Postdoctoral Scholar, Graduate Aeronautics Laboratory, California Institute of Technology, Pasadena, CA 91125. Member AIAA.

${ }^{\dagger}$ Holder of the Fred D. Durham Chair, Department of Aerospace and Ocean

Engineering, 215 Randolph Hall. Fellow AIAA.

Research Scientist. Member AIAA. and temperature profiles, Debieve et al. also developed a methodology to deduce the skin-friction coefficient and Stanton number. Good agreement with the velocity profiles, temperature profiles, skin friction, and Stanton number was obtained by Marineau et al. [3] with Reynolds-averaged Navier-Stokes (RANS) simulations. Menart et al. [4] studied the effect of volumetric and surface heating of a Mach $\overline{5}$ laminar boundary layer. The surface was heated with an electrical resistance heater mounted in the wall, and its effect was evaluated through changes in temperature and pressure inside the flowfield at different distances from the wall. Menart et al. found that the response of the surface heater is strongly influenced by the thermal properties of the plate. This is also expected for laser heating, which implies that the conservation equations for the flowfield and the solid target must be simultaneously solved. Such a problem in which the condition at the interface must be determined as a part of the solution is known at a conjugate heat transfer problem.

CHT problems are commonly found in aerospace applications such as turbine blade cooling [5]. Cooling of electronic components is also an active area of research involving CHT [6], which relates to the laser heating problem. The component is typically modeled as a surface or volumetric heat source immersed in a shear or channel flow [7,8], in which it is assumed that the heating does not affect the velocity field. This implies that the coupling is achieved through the solid and fluid energy equations that are uncoupled from the momentum equation. The extensive parametric study performed by Cole [7] showed the importance of the conjugate Peclet number for CHT problems as it directly influences the energy balance at the fluid-solid interface. The modeling of the beam-target interaction shares similarities with the cooling of electronic components, but displays an increase in complexity due to the supersonic flow and the high localized heat flux, which is expected to significantly affect the flowfield.

In our experiment, the surface temperature was measured using a combination of infrared thermography and thermocouples. Infrared thermography has previously been used for the visualization of turbulent structures in laminar-turbulent transition [9]. The developed experimental and computational methods could be used for the modeling and simulation of novel applications such as the heat

${ }^{\S}$ Data available online at http://www.boeing.com/defense-space/military/ abl/index.html [retrieved 2007]. 
exchanger thruster concepts [10], in which a heat exchanger absorbs laser energy and transfers it to the hydrogen, producing hot hydrogen that expands through a nozzle. Another possible application is the study of localized wall heating for boundary-layer control [11].

\section{Experimental Apparatus and Methods}

\section{A. Design Methodology}

The objective of the experiment was to investigate the effect of a supersonic flowfield over a laser-irradiated target, which is a difficult task due to the large temporal and spatial gradients over a very small area. Analytical methods and numerical simulations were used to design an experiment that is able to generate reliable measurements. To lower the experimental uncertainties, the convective cooling has to be maximized. This implies a maximization of the conjugate Peclet number $\Lambda_{L}$ first introduced by Cole [7] and the beam geometric ratio $w / L$, as shown by Marineau [12]. This introduces the following requirements: a thin turbulent boundary layer, a low solid thermal conductivity, and a thin plate and/or a large beam diameter.

To get a thin boundary layer, a flat plate must be inserted into the tunnel flow. It was determined by Marineau [12] using experimental data [13] for similar conditions that the boundary layer is fully turbulent at $0.09 \mathrm{~m}$ from the leading edge, and so the laser beam center was located after $0.1 \mathrm{~m}$ from the leading edge.

The effect of the material was investigated by Marineau [12], who found that stainless steel leads to a much greater conjugate Peclet number, due to its low thermal conductivity. Stainless steel also has the advantage of sustaining high temperature. AISI-303 stainless steel was chosen as the plate material.

The geometric ratio $w / L$ can be made large by increasing the beam width or by making the plate thin. To facilitate repeatability, we chose to avoid using any external optics. The thickness of the plate near the target center is fixed to $2.54 \mathrm{~mm}$.

\section{B. Overview of Components}

To our knowledge, no experimental study of the temperature distribution on a laser-heated target subjected to a supersonic flow is available in the literature. Experiments on a laser-heated ceramic disk have been performed at Oak Ridge Laboratory [14,15].

Experiments were performed in the Virginia Polytechnic Institute and State University $23 \times 23 \mathrm{~cm}$ blowdown supersonic wind tunnel with a Mach 4 nozzle, ambient total temperature, total pressure of $1.1 \times 10^{6} \mathrm{~Pa}$, and Reynolds number of $5 \times 10^{7} / \mathrm{m}$.

The main components of the test setup are depicted in Fig. 1 . As one can see, the system uses both infrared thermography and thermocouples (TC). Three data acquisition (DAQ) and control modules are present; each are linked to a different computer.

A drawing of the flat-plate model is shown in Fig. 2a. The plate contains two inserts that can be removed if damaged. The laser hits the upstream insert. The second insert downstream was used to mount a pitot tube. The strut-mounted plate can be located on the side wall or on the test-section floor plate, which allows taking schlieren or shadowgraph images.

The inserts were mounted on a 6.25-mm-thick plate. Because metals oxidize at high temperature, a protective coating must be used to insure good repeatability. The plate was painted using flat black paint (Pyromark 2500). This silicon-based paint has an absorptivity of $95 \% \pm 1 \%$ at the laser wavelength [16] and emissivity of 0.85 [17] and can sustain a temperature of $1350 \overline{\mathrm{K}}$.

A $300 \mathrm{~W}$, single-mode, continuous-wave ytterbium fiber laser (IPG Photonics) at $1080 \mu \mathrm{m}$ was used. The beam is Gaussian and its $4 \mathrm{~mm}$ diameter $\left(e^{-2}\right)$ does not significantly change with distance, as shown by Marineau [12]. The laser was controlled using a LabVIEW program developed in-house, and the collimator was mounted on an optical table on which it was positioned with 2 degrees of freedom (2DOF) using two translation stages, each with a resolution of $3 \mu \mathrm{m}$.

The midwave infrared camera (Indigo Merlin) has a $256 \times 320$ cell Indium antimonide detector (sensitive between 3 and $5 \mu \mathrm{m}$ ), a 12-bit dynamic range, and a maximum frame rate of $50 \mathrm{~Hz}$. An ND2 filter avoids saturation for high-temperature measurements. For each integration time, filter, and lens combination, a radiometric calibration was performed by comparing the digital output of the camera with the known temperature of a uniform blackbody. The variability of each cell was corrected using a nonuniformity correction table (NUC). The NUCs were generated during the calibration process. The precision of the system is therefore dependent on the uniformity of the blackbody. Six calibrations, NUC, were performed for a $25 \mathrm{~mm}$ lens by the manufacturer and checked inhouse using a blackbody. The integration times and temperature ranges corresponding to each NUC are shown in Table 1. Positioning the lens at about 6 in. from the target gave a spatial resolution of approximately $0.240 \mathrm{~mm}$.

Because the temperature range due to heating is larger than the dynamic range of the camera, many runs must be repeated to get the totality of the temperature field. For a power of $81 \mathrm{~W}$, the NUC0, NUC2, NUC3, NUC4, and NUC5 were used, such that 5 runs were required. At each instant, the images must be combined to give a single image. Also, each pixel must be mapped to a given physical coordinate. A data-reduction program written in MATLAB was designed to perform those tasks; further details are found in [12].

Two type-K surface temperature thermocouples [18] (Medtherm Corporation) were used to make surface measurements. One

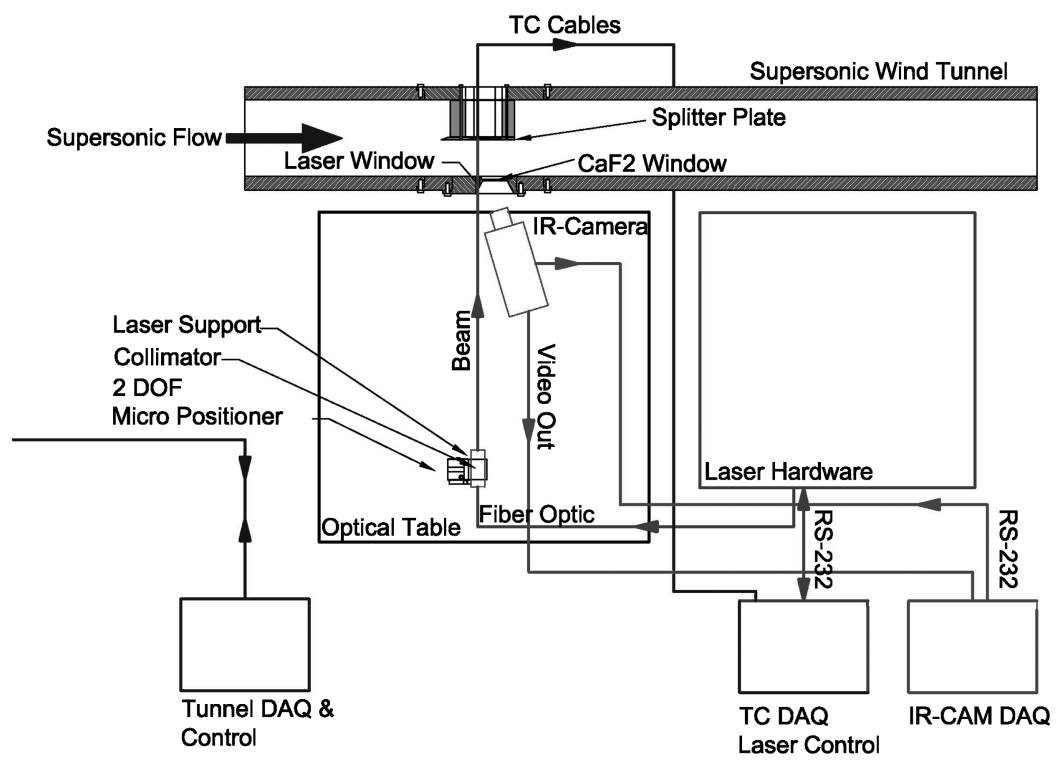

Fig. 1 Overall experimental layout. 


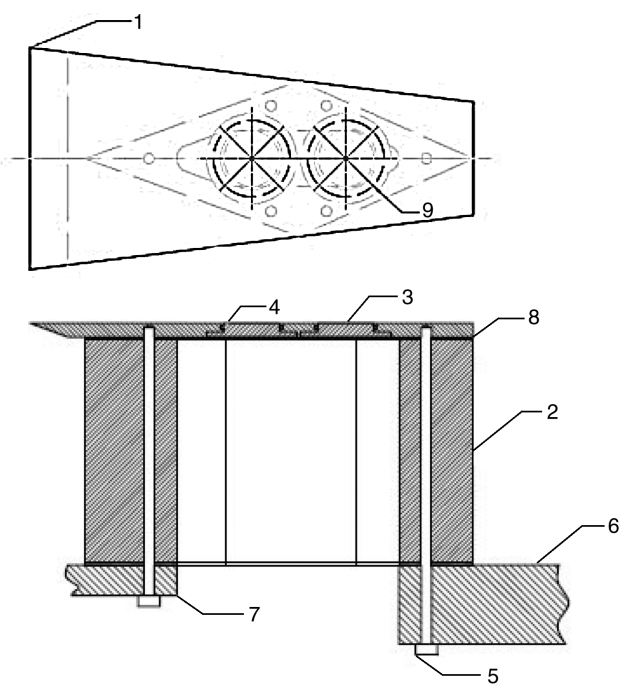

a) Flat-plate model

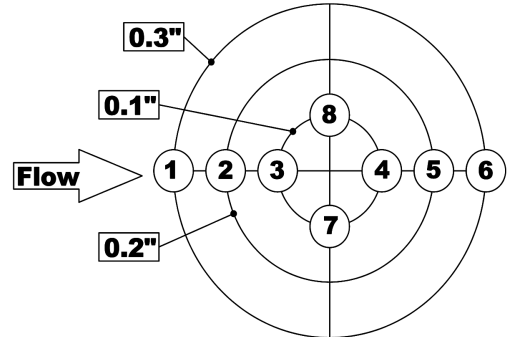

b) Backside thermocouples position

Fig. 2 Flat-plate model and backside thermocouple positions.

(0.381 $\mathrm{mm}$ in diameter) was located at the center of the heated insert. The other ( $1.549 \mathrm{~mm}$ in diameter) was located upstream to provide an accurate wall temperature boundary condition for our numerical simulations. A fast response time is made possible by the $2-\mu \mathrm{m}$-thick junction formed by a vacuum-deposited metallic coating over the sensing end of the probe. During the test, the center thermocouple developed problems. Because the surface temperature was kept lower than the thermocouple maximum operating temperature, we believe that the failure can be attributed to a reaction between the thin thermocouple junction and the silicone-based paint.

Eight type-K thermocouples (Omega SRTC-TT-K-40-36) made of $0.076 \mathrm{~mm}$ (gauge 40) wires were fixed to the backside of the plate using high-temperature cement (Omegabond 400). Positions are shown in Fig. 2b. Such fine wires enable precise measurements and fast response time. A 16-bit analog-to-digital converter (National Instruments 6036E) with multiplexer (AMUX-64T) was used for thermal data acquisition.

A glass window (BK7) of $12.7 \mathrm{~mm}$ in diameter with an antireflection coating for the laser wavelength was mounted on the tunnel wall, allowing the laser access to the target. A calcium fluoride $\left(\mathrm{CaF}_{2}\right)$ window was used to view the target. Calcium fluoride is ideal for midwave infrared measurements, because it possesses a high and constant transmitivity between 3 and $5 \mu \mathrm{m}$ as well as an intrinsically low reflectivity. Optical properties of both windows are found in [12].

\section{Uncertainty Analysis}

To cancel the bias errors, the temperature difference between the flow-off and flow-on cases was recorded. For that strategy to be effective, the tests must be highly repeatable. Repeatability was quantified and the uncertainty of temperature difference between the flow-off and flow-on cases was determined for both the thermographic and thermocouples measurements. A detailed uncertainty analysis was performed by Marineau [12]. The uncertainty on the temperature is equal to $5.35 \%$ as computed by considering the uncertainty in the laser power, the surface absorptivity, emissivity, and the calibration error. The uncertainty on the thermocouple measurement is equal to $3.8 \%$. To evaluate the uncertainty on the temperature difference, the random error was quantified using the backside temperature repeatability for three runs. Student's $t$-distribution was used to compute the uncertainty of the mean considering the limited number of samples. The effects of the alignment and timing errors are computed using an analytical solution developed by Marineau and combined using the root-meansquare formula. For the thermocouple, the repeatability from six runs was used to compute the random error. The uncertainties on the temperature difference for the infrared camera and the thermocouple are plotted in Figs. $3 \mathrm{a}$ and $3 \mathrm{~b}$.

We notice that uncertainty is a function of position and time. For the infrared measurements at the center on the surface, the uncertainty is smaller, due to the absence of the positioning error. The positioning error is approximately equal to zero at the center, because it corresponds to a maximum in the temperature distribution. On the surface, the positioning error is the greatest at $1 \mathrm{~mm}$ from the center, due to the large radial temperature gradient. From that position, the positioning error progressively decreases with distance, such that away from the center the uncertainty is mainly due to the random errors. The uncertainty is greater for thermocouples 3 and 8 , which are the closest to the center. For most thermocouples, the uncertainty increases with time.

\section{Experimental Results}

\section{A. Flow Survey}

The boundary-layer thickness was estimated with a schlieren image, and the shock on the plate leading edge was visualized best by using the Shadowgraph method. Figure 4 a shows that the boundary layer is turbulent over the plate as eddies are identified. The boundary-layer thickness is equal to $4 \mathrm{~mm}$ at the measurement station. Using the shock angle shown in Fig. $\underline{4 b}$, the edge Mach number $M_{e}$ is estimated to 3.8. A value of $M_{e}=\overline{3} .75$ was obtained using static and pressure measurements.

\section{B. Test Matrix}

The test matrix is presented in Table 2. Different values of laser power were used to evaluate the effects of the power on the temperature difference. At each power, two tests were made: one with the flow on and the other with the flow off. For the flow-on case, the laser was turned on after the tunnel started and the flow reached a steady state. For the flow-off case, the plate was heated at the same power but without the supersonic flow. The cooling effect was seen by subtracting the flow-on temperature from the flow-off 


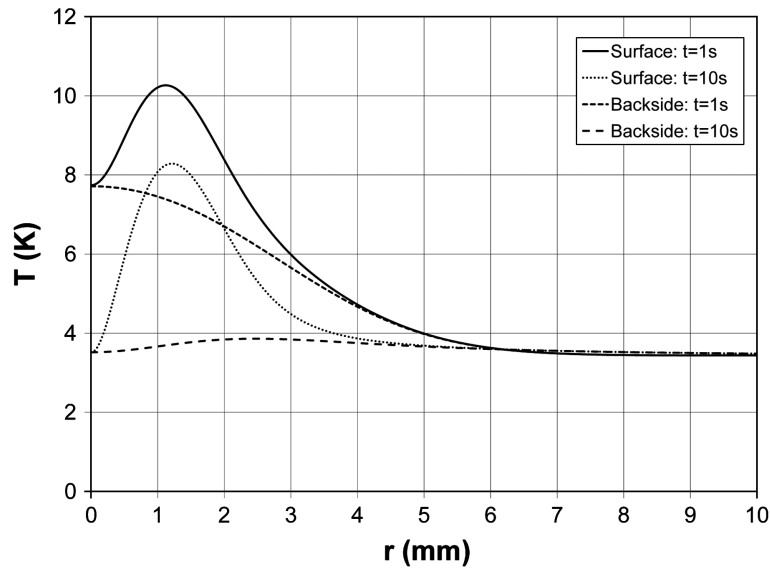

a) Infrared camera

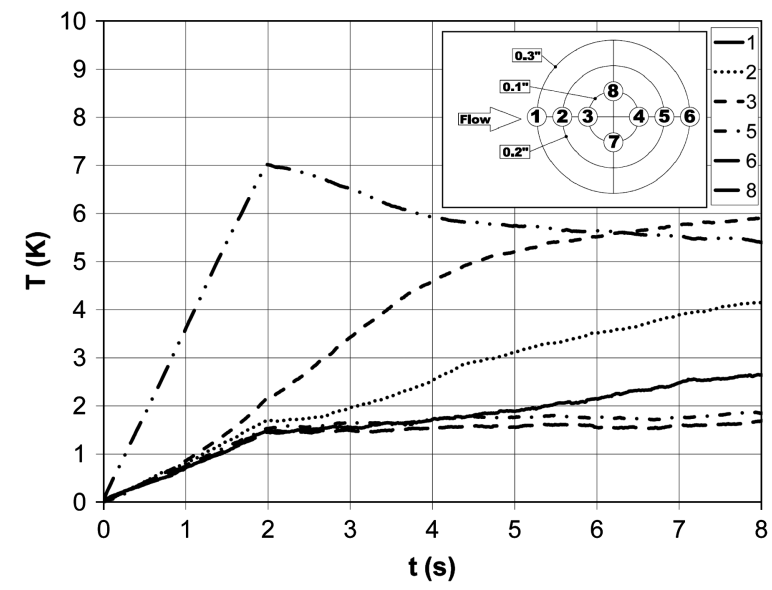

b) Thermocouples

Fig. 3 Uncertainty estimates in the temperature difference measured by the a) infrared camera on the surface and backside as a function of distance for $t=1$ and $10 \mathrm{~s}$ and $\mathrm{b}$ ) thermocouples as a function of time.

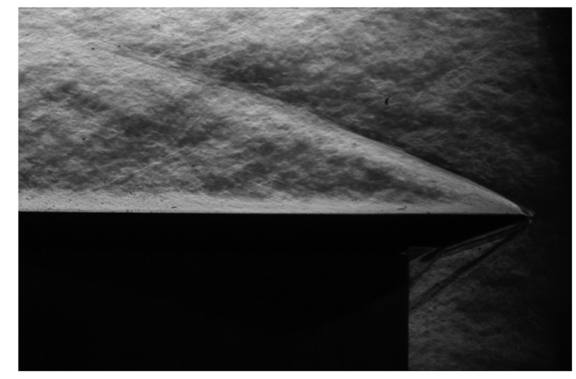

a) Boundary-layer schlieren

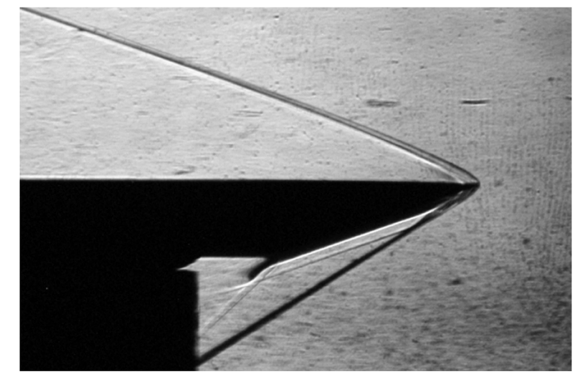

b) Leading-edge shock shadowgraph

Fig. 4 Flow visualization used to determine the edge Mach number and boundary-layer thickness.

temperature. No infrared measurements were made at $101 \mathrm{~W}$, and only the high-temperature calibration was used at $120 \mathrm{~W}$.

\section{Thermocouple Results}

The backside temperature was measured for 6 runs at $81 \mathrm{~W}$. Overall, repeatability was good, as the run-to-run difference was less that $5 \mathrm{~K}$. Figure 5 a shows the temperature increase for the flow-on test. Slight asymmetry was found in the temperature distribution for the flow-off case, which is most likely due to the slight laser misalignment. However, because the alignment was kept the same for the flow-on and flow-off cases, misalignment should have a minor effect when the temperature difference between the flow-off and flow-on cases is taken. The temperature difference between the flowoff and flow-on cases for run 1 is plotted in Fig. 5b. The greatest cooling is seen close to the center for thermocouples 3 and 8 . The maximum cooling is between 20 and $25 \mathrm{~K}$ at the center. We clearly notice some asymmetry by looking at the thermocouple pairs 1-6 and 2-5. The difference between thermocouples 2 and 5 reached almost $10 \mathrm{~K}$ at $t=10 \mathrm{~s}$. As expected, thermocouple 5 cools less than thermocouple 2, because heat is convected downstream. The same situation arises when comparing thermocouples 1 and 6 , as the difference in cooling is close to $10 \mathrm{~K}$ at $t=10 \mathrm{~s}$. Clearly, a steady state was not achieved during the run, as the temperature difference for all the thermocouples keeps increasing.

Table 2 Test matrix

\begin{tabular}{lcc}
\hline \hline Laser power, $W$ & Maximum intensity, $\mathrm{W} / \mathrm{cm}^{2}$ & Time on, $\mathrm{s}$ \\
\hline 65 & 1035 & 10.8 \\
81 & 1289 & 10.8 \\
101 & 1607 & 10.8 \\
120 & 1910 & 10.8 \\
\hline \hline
\end{tabular}

\section{Infrared-Camera Results}

Figure 6 a shows the surface temperature contours for the flow-on and flow-off cases at $10 \mathrm{~s}$ for $81 \mathrm{~W}$ of power. The flow is directed from left to right. The upper half of each contour plot corresponds to the flow-off case, and the lower part corresponds to the flow-on case. Some disturbance in the temperature contours can be seen on the upper part around $r=4 \mathrm{~mm}$, due to paint damage. Over the damaged area, the emissivity is less, which explains the disturbance. For the flow-off case the temperature reaches $1175 \mathrm{~K}$ after $10 \mathrm{~s}$. The maximum temperature reaches $1115 \mathrm{~K}$ after $10 \mathrm{~s}$ for the flow-on case. Maximum cooling was achieved at the center. More cooling can be seen upstream of the heated spot compared with downstream, where the isolines almost coincide.

Figure $6 \mathrm{~b}$ shows the temperature distribution on the backside at $7 \mathrm{~s}$. The asymmetry is difficult to notice from the temperature plots. The maximum temperature at the center reaches $675 \mathrm{~K}$ after $8 \mathrm{~s}$ for the flow-off case and $650 \mathrm{~K}$ for the flow-on case.

The asymmetry in cooling is better seen with the temperature difference between the flow-on and flow-off cases. The surface temperature difference is found in Fig. 7, and Fig. 8 gives the backside temperature difference. On the surface, a region of increased cooling is seen upstream, which is followed by a region of reduced cooling right before the center. At the center, the cooling reaches about $50 \mathrm{~K}$ after $9 \mathrm{~s}$. At $t=1 \mathrm{~s}$, the maximum temperature difference reaches $60 \mathrm{~K}$. As seen in the uncertainty analysis, the uncertainty is greater at a small value of time, due to the increase in the timing error from the large time rate of change of the temperature. Also, the flow-on and flow-off cases are on a different calibration for that value of time, such that the bias error does not cancel. It is therefore believed that the temperature difference is somewhat overpredicted at $t=1 \mathrm{~s}$.

The asymmetry is also seen on the backside; clearly, more cooling occurs upstream than downstream. The effect of the mismatching calibration can be seen on all the backside images. For instance, at 


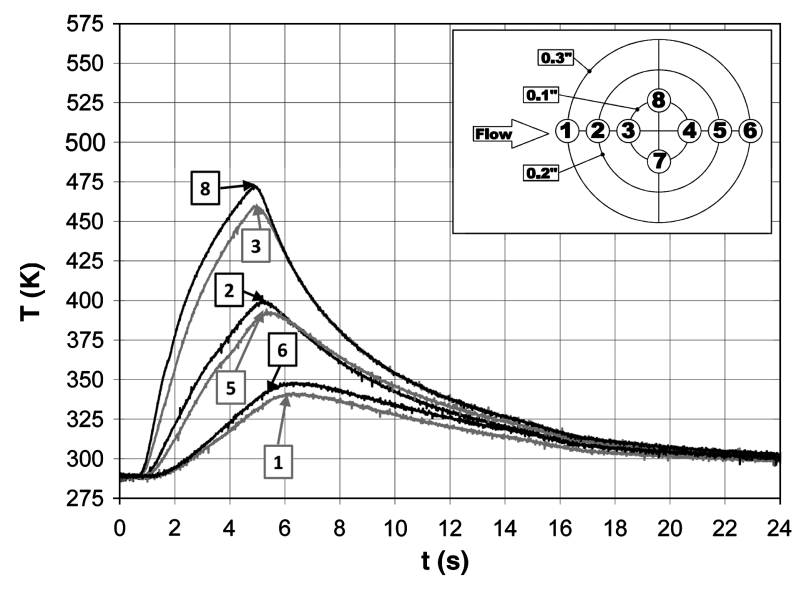

a) Backside temperature flow-on at $\mathrm{P}=81 \mathrm{~W}$

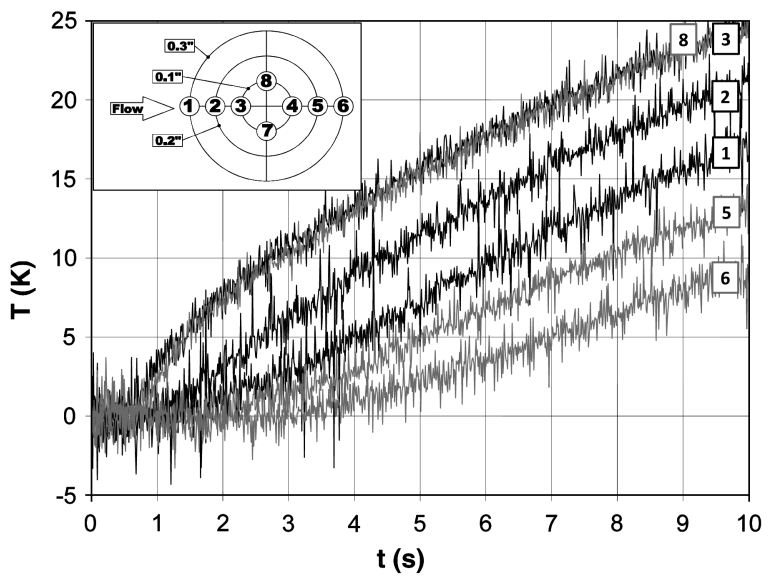

b) Backside temperature difference at $P=81 \mathrm{~W}$

Fig. 5 Backside thermocouple temperature measurements for run 1 at $P=81 \mathrm{~W}$.

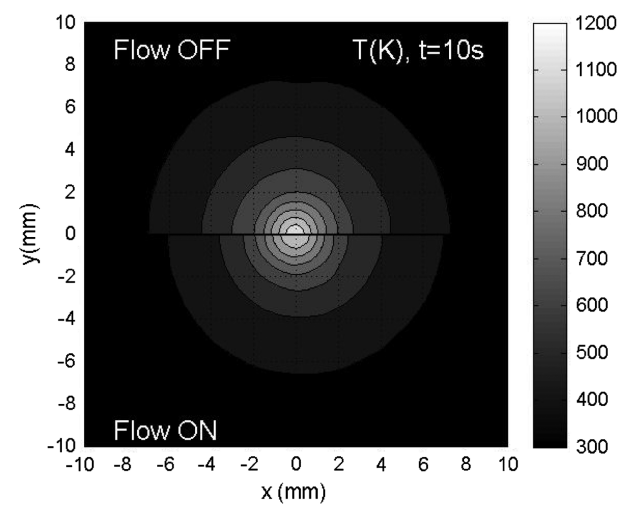

a) Surface temperature at $\mathrm{t}=\mathbf{1 0 \mathrm { s }}$

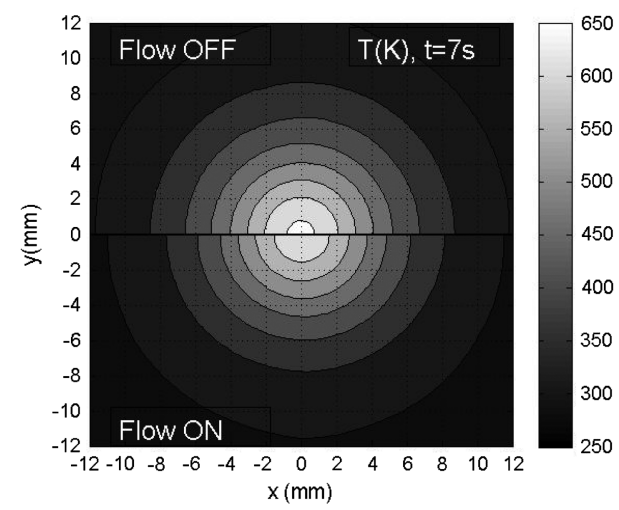

b) Backside temperature at $\mathrm{t}=7 \mathrm{~s}$

Fig. 6 Temperature contours from the infrared camera for $P=81 \mathrm{~W}$ with flow going from left to right; flow off (top) and flow on (bottom).

$t=1$, a disk of high cooling is seen at $r=4 \mathrm{~mm}$. This cooling is not real, as it is produced from the noncancellation of the bias error for mismatching integration time. As time increases, the radius of the disk increases. Data over that area must therefore be discarded.

The $120 \mathrm{~W}$ case displayed the greatest difference between the flow-off and flow-on cases. The maximum surface temperature is plotted as a function of time for flow on and flow off in Fig. 9. After $10 \mathrm{~s}$, a temperature difference of $90 \mathrm{~K}$ was measured. As shown in Fig. 9, the difference between the flow-off and flow-on cases remains almost constant between 6 and $10 \mathrm{~s}$. For times below $2 \mathrm{~s}$, the signal is too weak to get an accurate measurement (as only the high-temperature calibration was used). Figure 9 also shows that convective cooling significantly changes the time required to reach a given temperature. For example, for the flow-off case, only $5 \mathrm{~s}$ are required to reach $1500 \mathrm{~K}$, compared with $10 \mathrm{~s}$ for the flow-on case.

\section{E. Experimental Results Summary}

A summary of the experimental results is shown in Table 3. A linear relation is found between the maximum surface temperature and the laser power. Figure 10 shows that the linearity between the maximum surface temperature difference and the maximal surface temperature increase at $t=10 \mathrm{~s}$ (which is proportional with the laser power), where both are normalized with the recovery temperature. The slope, equal to $7 \%$, should give a good approximation of the heat flux reduction due to the flow, as the cooling is expected to be proportional to the temperature increase, which varies by only $15 \%$ between $t=3$ to $10 \mathrm{~s}$. In Fig. 11, the flow-off results are scaled by multiplying the maximum surface temperature increase by $93 \%$. The scaled flow-off results closely match the flow-on results for a dimensionless time $\left(t^{*}=t / \tau=t \alpha_{s} / w^{2}\right)$ greater than 3, which corresponds to $t=1.1 \mathrm{~s}$, where $w$ and $\alpha_{s}$ are the thermal diffusivity of the solid and the beam waist.
On the backside, a similar temperature difference is observed between the 65 and $81 \mathrm{~W}$ cases. More asymmetry was found at $81 \mathrm{~W}$ compared with $65 \mathrm{~W}$. This was observed on the surface and backside with both the infrared camera and thermocouple measurements. For the $81 \mathrm{~W}$ case, a region of increased cooling is seen upstream, where a coherent structure clearly appears. More testing at intermediate powers could help explain why such a difference is seen between the 81 and $65 \mathrm{~W}$ cases.

\section{Computational Studies}

\section{A. Computational Model}

GASP version 4.3 (AeroSoft, Inc.) was used for this study. The integral form of the time-dependent RANS equations in three dimensions were solved. Roe flux-difference splitting [19] was used to compute the inviscid flux with third-order spatial accuracy using the MUSCL reconstruction [20]. The viscous flux was computed with second-order spatial accuracy using a central difference. Unsteady solutions were obtained using the dualtime-stepping method with third-order accuracy. A solver for the three-dimensional heat-conduction equation was added to GASP to perform conjugate heat transfer problems. The conjugate heat transfer algorithm, which insured continuity of the temperature and conservation of energy at the fluid-solid interface, was described and validated for high-speed flow by Marineau et al. [21].

The freestream Mach number was set to 3.75, the static temperature was $77.4 \mathrm{~K}$, and the static pressure was $9770.5 \mathrm{~Pa}$. A boundary-layer profile from a flat-plate simulation run up to the measured thickness was specified at the inlet with a pointwise boundary condition. 


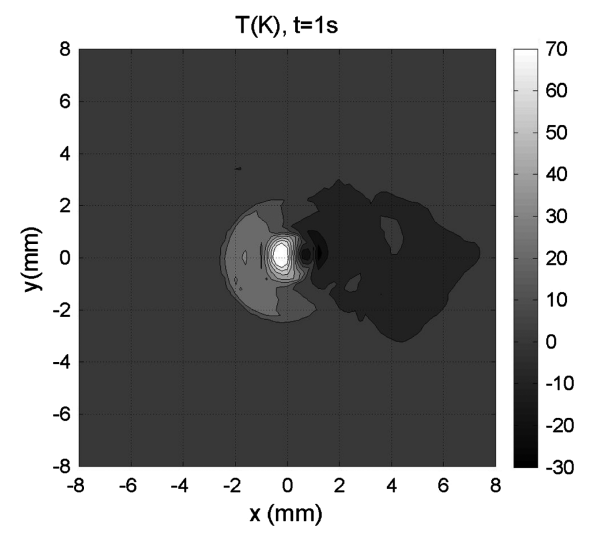

a) $t=1 s$

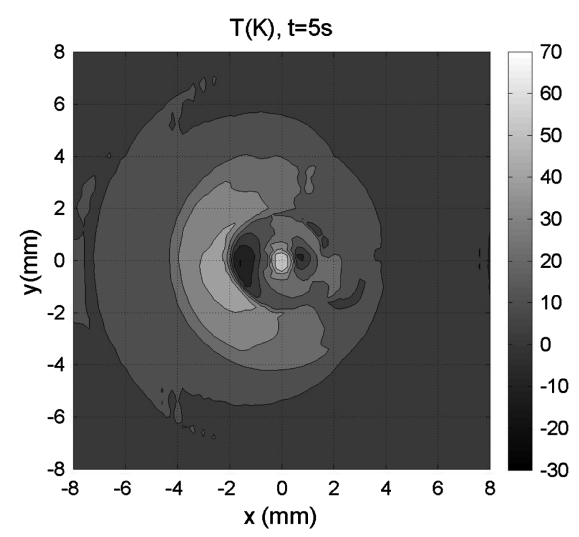

d) $\mathbf{t}=\mathbf{5 s}$

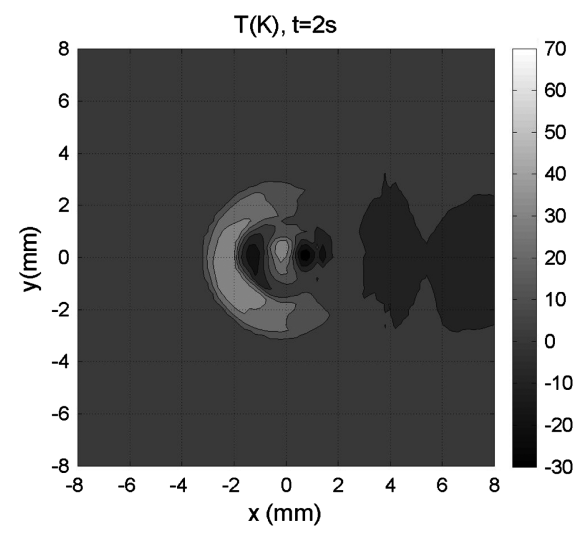

b) $t=2 s$

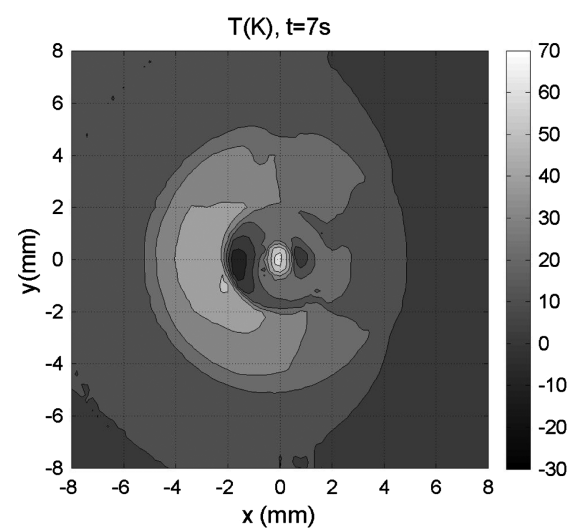

e) $\mathbf{t}=7 \mathrm{~s}$

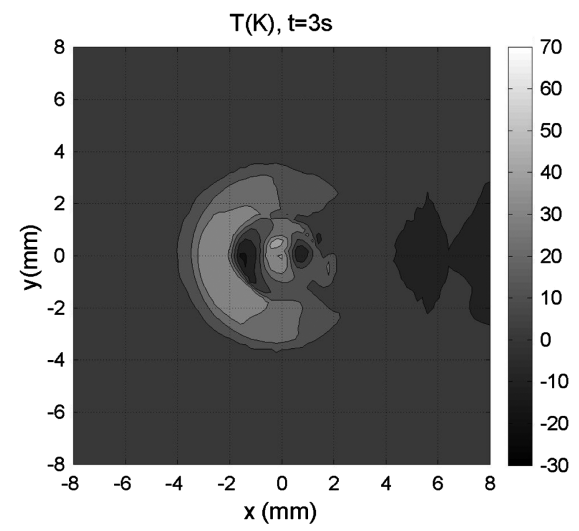

c) $\mathbf{t}=3 \mathrm{~s}$

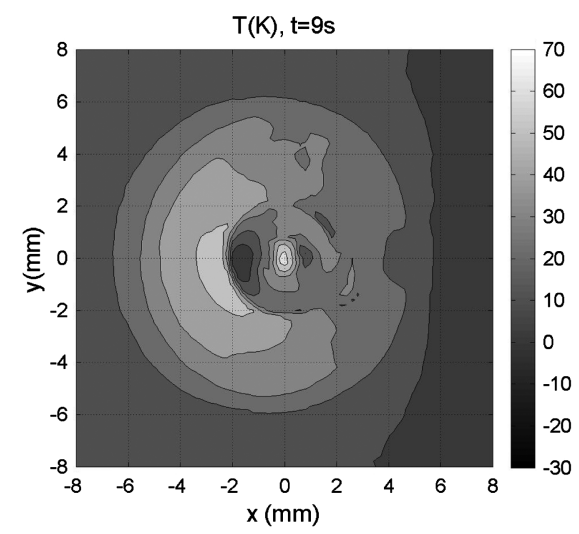

f) $\mathrm{t}=9 \mathrm{~s}$

Fig. 7 Surface temperature difference for $P=81 \mathrm{~W}$ with the flow going from left to right.

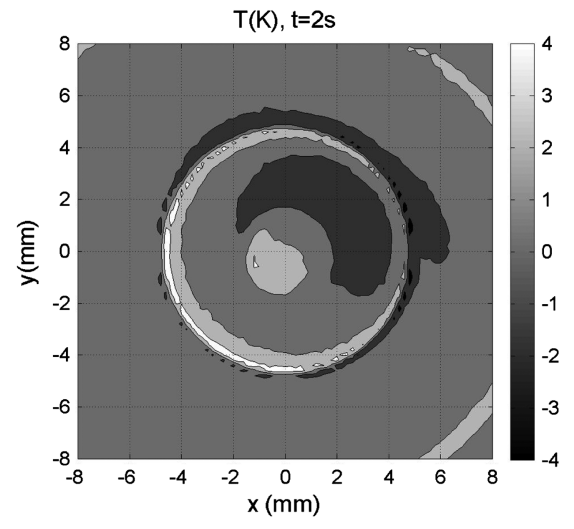

a) $\mathbf{t}=\mathbf{2 s}$

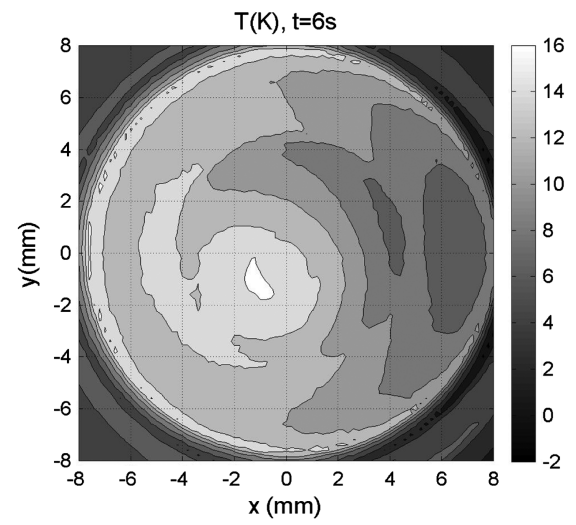

d) $t=6 s$

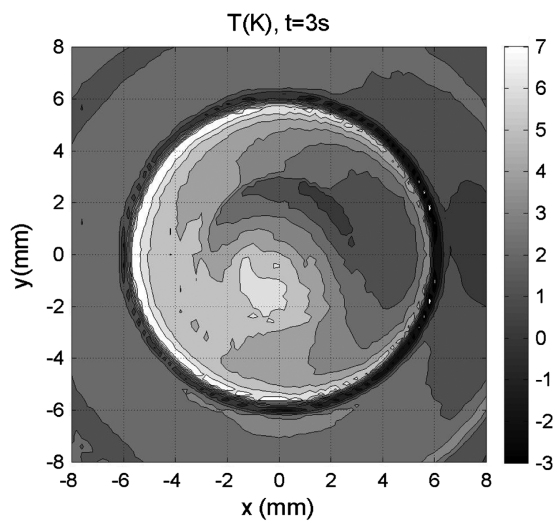

b) $\mathbf{t}=\mathbf{3 s}$

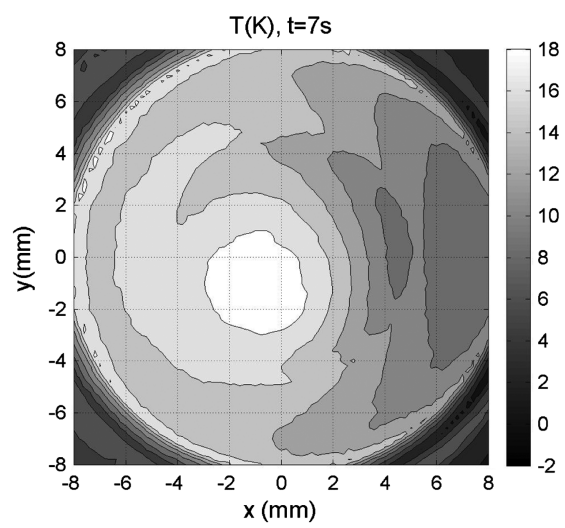

e) $\mathrm{t}=7 \mathrm{~s}$

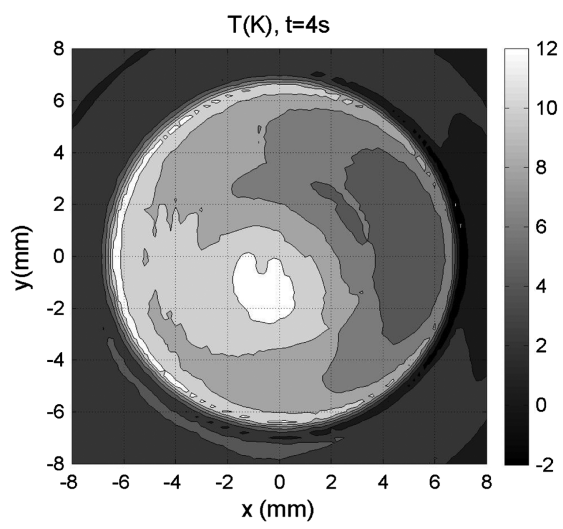

c) $\mathbf{t}=4 \mathrm{~s}$

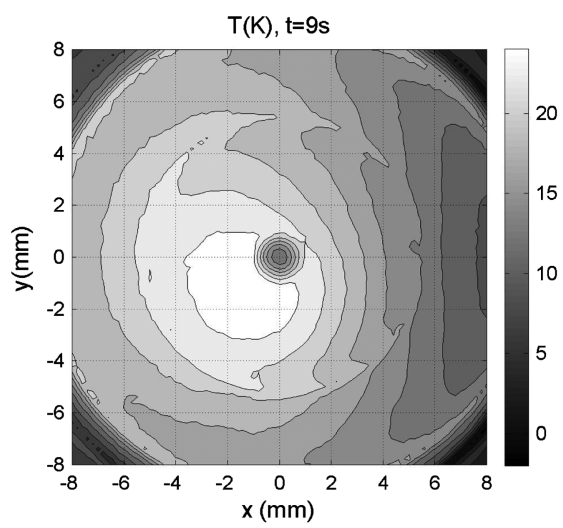

f) $\mathrm{t}=9 \mathrm{~s}$

Fig. 8 Backside temperature difference for $P=81 \mathrm{~W}$ with the flow going from left to right. 


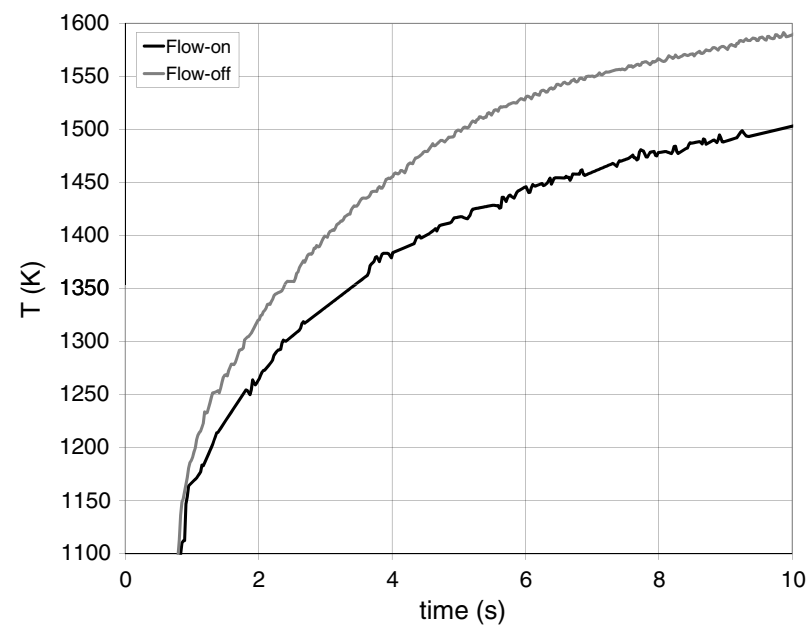

Fig. 9 Maximum surface temperature for flow-on and flow-off conditions at $P=120 \mathrm{~W}$.

To simulate the experiment, the fluid portion of the mesh was solved with a constant wall temperature until a steady state was achieved. The steady-state solution became the $t=0$ solution for the time-accurate run. That way, modeling of the tunnel's very complex starting process is avoided. This simplification is valid, as the change in temperature is small during the short startup process. The steadystate problem is solved using an infinite time step such that the system is solved with a Newton iteration [12] and converged to a global residual of $10^{-12}$. Ten iterations are used on the inner problem.

The laser takes $1 \mathrm{~s}$ before reaching its selected power. The startup is modeled using the following function:

$$
g(r, t)= \begin{cases}g(r) \sin \left(\frac{\pi t}{2 T_{s}}\right) & 0 \leq t \leq T_{s} \\ g(r) & t>T_{s}\end{cases}
$$

where $r$ is the distance from the beam center; the startup time of the laser, $T_{s}$ equals $1 \mathrm{~s}$; and $g(r)$ is the intensity (heat flux per unit area) distribution for a Gaussian beam, defined as

$$
g(r)=I_{0} \exp \left(-r^{2} / w^{2}\right)=\frac{P}{\pi w^{2}} \exp \left(-r^{2} / w^{2}\right)
$$

where $w$ is the radius at which the intensity decreases by a factor equal to $e^{-1}, I_{0}$ is the maximum surface intensity, and $P$ is the absorbed power. Simulations were performed at 65 and $81 \mathrm{~W}$ of absorbed power. The surface intensity was specified with a pointwise boundary condition.

The time-accurate simulation was performed using a physical time step of $3 \times 10^{-4} \mathrm{~s}$. Within the dual-time-stepping formulation, 10 inner cycles were performed for each time step. The inner pseudo time step is set to infinity (i.e., $10^{15}$ ). All cases were solved with the one-equation Spalart-Allmaras turbulence model. However, to assess the sensitivity to turbulence modeling, some cases were solved using Wilcox's $k-\omega$ and Menter's shear-stress-transport turbulence models. It was noticed that turbulence modeling has a small effect on the maximum surface temperature.

The grid was generated with Gridgen. Only one-half of the geometry is modeled, due to symmetry. Three grid densities (coarse, medium, and fine) were generated, and the generalized Richardson

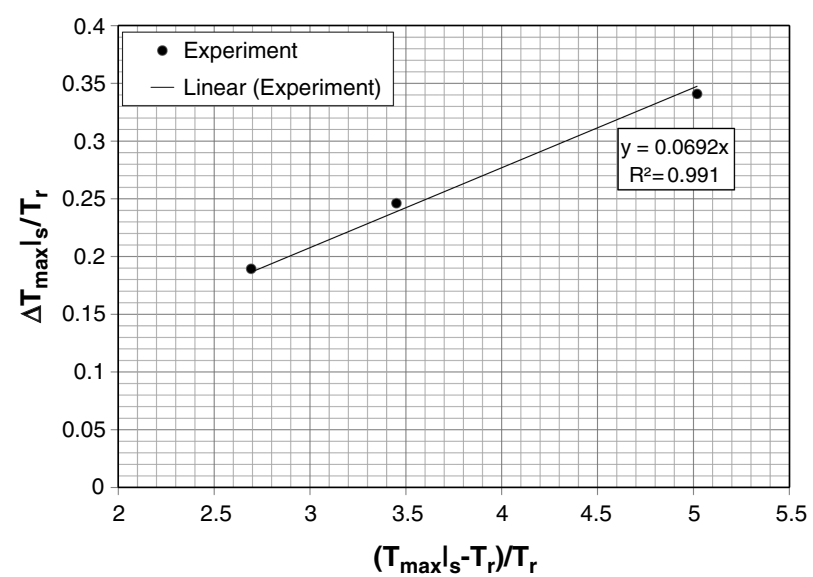

Fig. 10 Linearity between maximum temperature difference and maximum temperature increase on the surface.

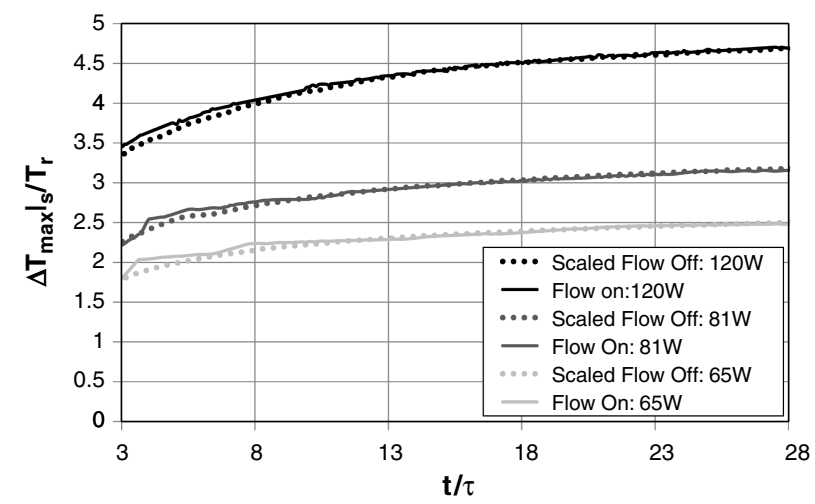

Fig. 11 The flow-off maximum temperature increase multiplied by $93 \%$ scales with the flow-on solution for $t^{*} \geq 3$.

extrapolation method [22] was used to compute the spatial and temporal discretization errors, which were combined into the total discretization error using the root-mean-square formula. With flow on, the total relative error on the maximum temperature for the medium grid is $2.2 \%$, compared with $1.2 \%$ with flow off. The uncertainty on the temperature difference [(flow off) - (flow on)] at the target center is $5.9 \%$ (of the temperature difference) for the medium grid. The details of the grid and time convergence study are presented by Marineau [12].

The medium grid for the fluid domain is shown in Fig. 12. An $\mathrm{H}-\mathrm{C}-\mathrm{H}$ topology was used, allowing localized clustering. The conjugate heat transfer boundary condition was used at the fluidsolid interface. An adiabatic boundary condition was used on the edge and on the backside of the solid. Convective and radiative boundary conditions on the backside were tried, but the effect on the temperature was insignificant (less that $0.01 \mathrm{~K}$ ). In the fluid, hyperbolic tangent clustering was used in the $z$ direction to properly capture the boundary layers. For the medium grid, more than 40 points are located in the boundary layer. The center of the first cell of the wall is located at $2.5 \times 10^{-6} \mathrm{~m}$ from the wall to get a value of $y^{+}$ of 0.5 . In the solid, hyperbolic tangent clustering was also used in the $z$ direction.

Table 3 Results summary for temperature and temperature difference of surface $\left.\right|_{s}$ and backside $\left.\right|_{b}$

\begin{tabular}{lcccccccc}
\hline \hline$P, \mathrm{~W}$ & $\left.T_{\max }\right|_{s}, \mathrm{~K}$ & $t, \mathrm{~s}$ & $\left.\Delta T_{\max }\right|_{s}, \mathrm{~K}$ & $t, \mathrm{~s}$ & $\left.T_{\max }\right|_{b}, \mathrm{~K}$ & $t, \mathrm{~s}$ & $\left.\Delta T_{\max }\right|_{b}, \mathrm{~K}$ & $t, \mathrm{~s}$ \\
\hline 65 & 975 & 10 & 50 & 9 & 600 & 10 & 27 & 10 \\
81 & 1175 & 10 & 65 & 10 & 675 & 10 & 25 & 8 \\
101 & -1589 & -10 & -90 & -10 & - & - & 12 & 4.5 \\
120 & 1589 & - & - & - & - \\
\hline \hline
\end{tabular}




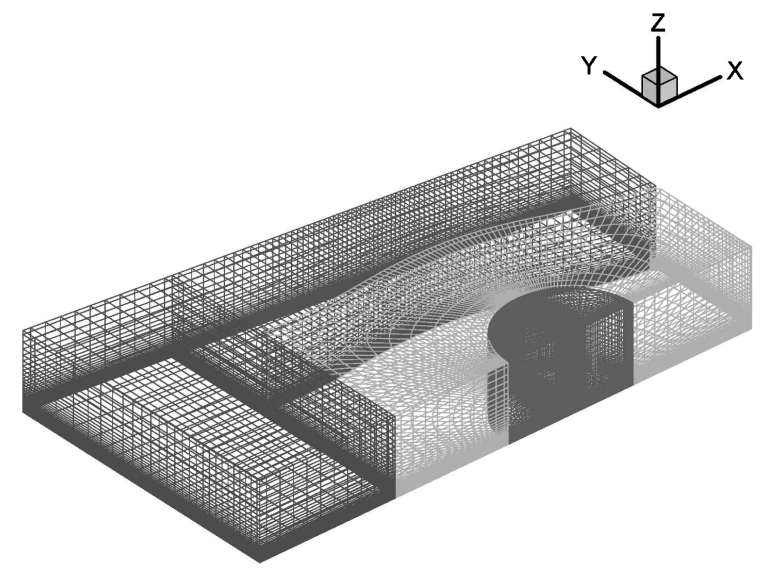

Fig. 12 Medium grid for the fluid domain composed of four blocks. Half of the geometry is modeled due to symmetry.

\section{B. Computational Results}

The use of computational fluid dynamics (CFD) makes it possible to visualize the change produced by the wall heating on the flowfield. Results for $65 \mathrm{~W}$ of laser power are presented in Fig. 13. Similar results were observed for the $81 \mathrm{~W}$ case. Moving away from the wall, note that the temperature contours get elongated in the flow direction, as shown in Fig. 13. The temperature disturbance is localized in a thin near-wall region, such that for a maximum wall temperature of $900 \mathrm{~K}$, the maximum temperature in the flow decreases to $400 \mathrm{~K}$ at only $0.1 \mathrm{~mm}$ from the wall. Other flow variables are also affected by the heated region. A reduction of $60 \%$ in the fluid density is observed and an increase in laminar viscosity exceeding $100 \%$ is observed. The vorticity in the $y$ direction is significantly decreased due to fluid dilation. Detailed results are given by Marineau [12].

The pressure disturbances emanating from the heated region of the wall get convected downstream along Mach lines, as shown in Fig. 14b. The intensity of the disturbances is progressively reduced with increasing distance from the wall, due to three-dimensional effects. Figure 14 a shows the streamlines and $w$-velocity contours in the symmetry plane. To improve the visualization of the flow features, the scale is stretched by a factor of 20 in the $z$ direction. Clearly, the heated spot acts as a bump, which slightly turns the flow upward. Very close to the wall, the flow is turned downward behind the bump, as a region of negative $w$ velocity is seen. The positive disturbance in the $w$-velocity component is convected along Mach lines, whereas the negative disturbance is quickly damped out such that it remains near the wall.

\section{Comparison of Experiment and Computation}

Comparisons between the experiment and the computations are presented for the $P=81 \mathrm{~W}$ case in Figs. 15-19. Figures $15 \mathrm{a}$ and $15 \mathrm{~b}$ show the temperature profiles on the target surface with flow off and flow on. Comparisons on the backside are found in Figs. 16a and 16b.

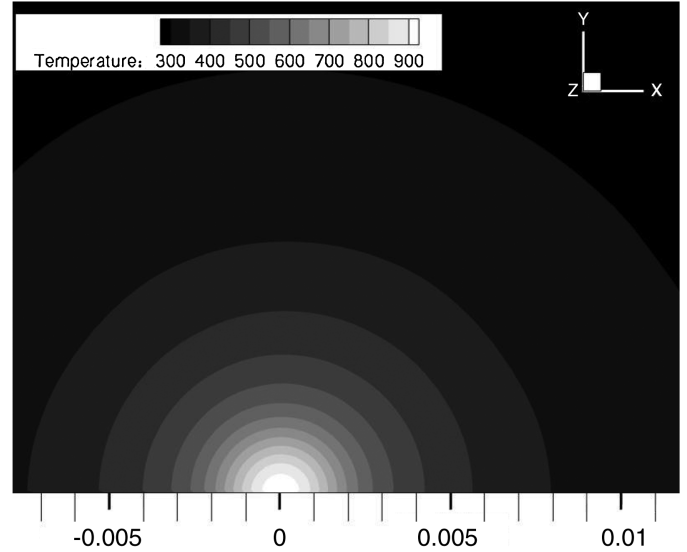

a) Wall

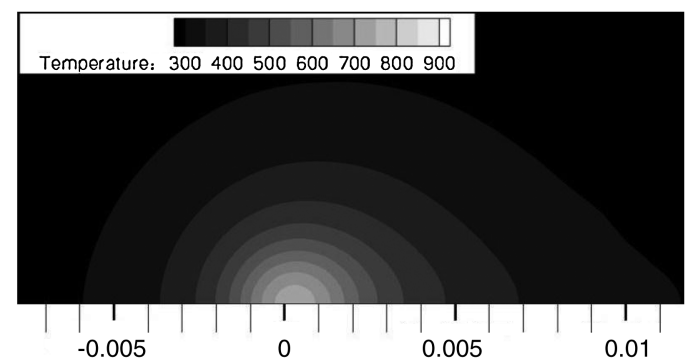

b) $z=0.04 \mathrm{~mm}$

Fig. 13 Predicted temperature contours in the fluid in the $x-y$ plane. Flow is from left to right. Dimensions are in meters.

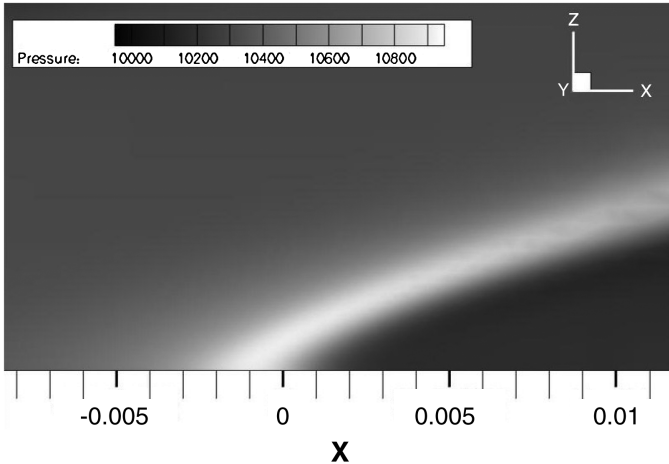

a) Pressure iso-contours

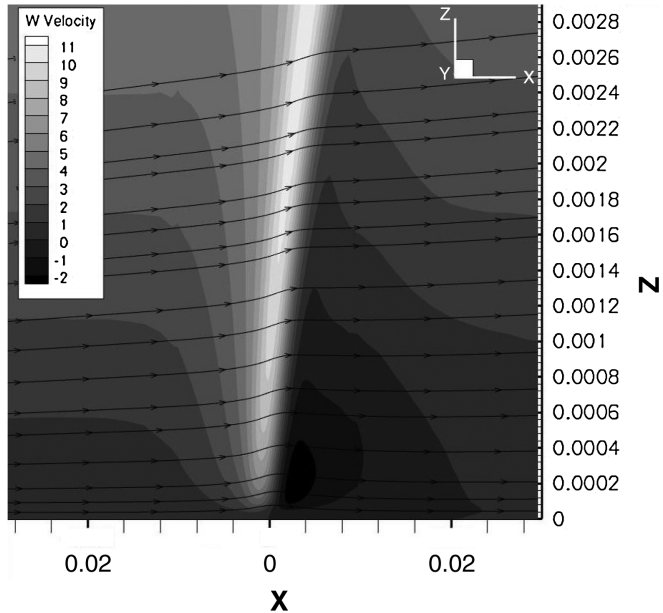

b) Streamlines

Fig. 14 CFD results: a) pressure isocontours at $t=10 \mathrm{~s}$ for $P=65 \mathrm{~W}$ (a high-pressure region is created upstream of the heat spot, and a low-pressure region is created downstream) with dimensions are in meters and b) streamlines in the $x-z$ plane at $t=10 \mathrm{~s}$. The heat source acts like a bump. 


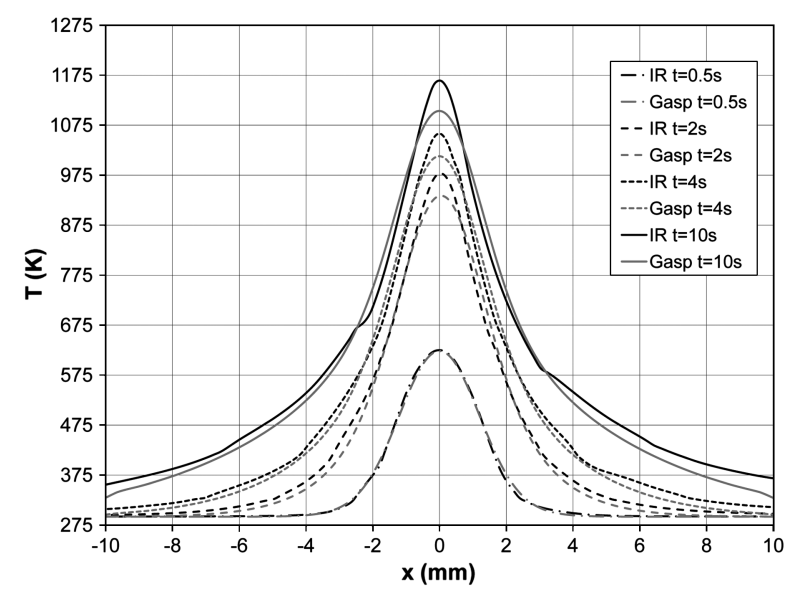

a) Flow-off

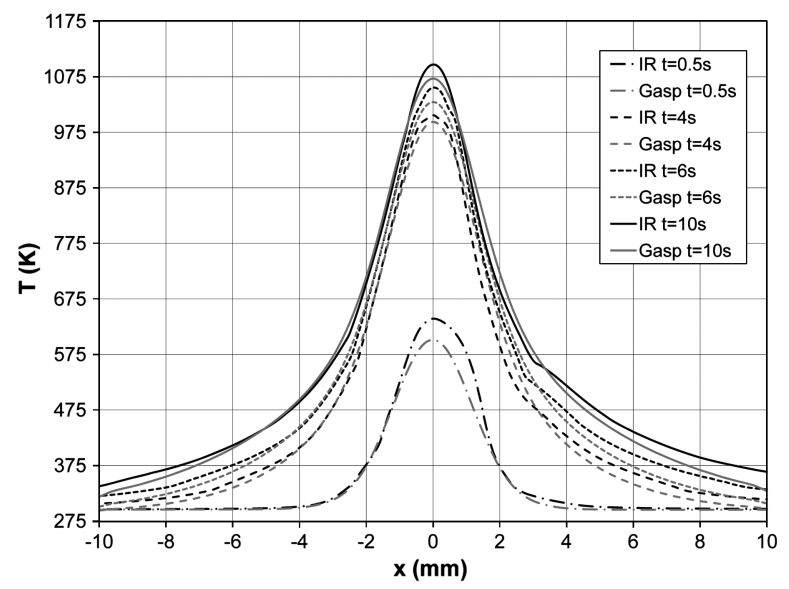

b) Flow-on

Fig. 15 Comparison between infrared measurement and computed surface temperature as a function of time at $P=81 \mathrm{~W}$.

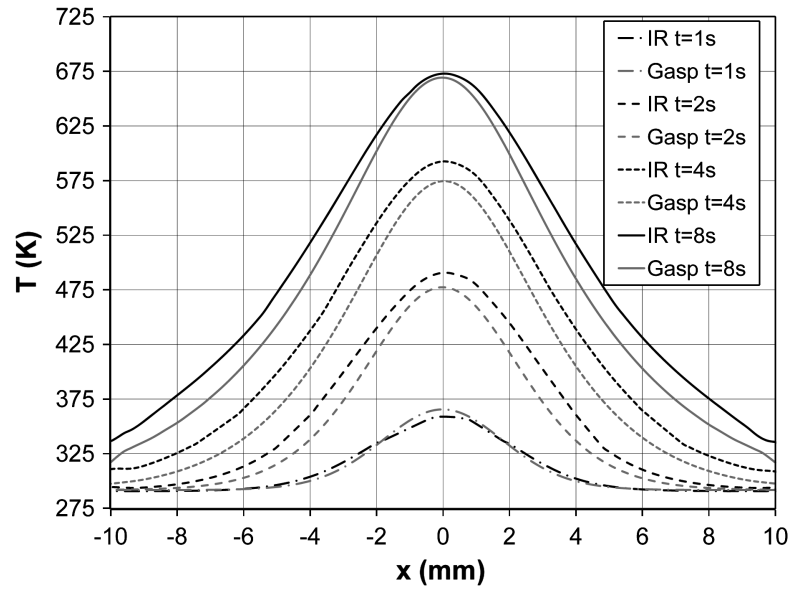

a) Flow-off

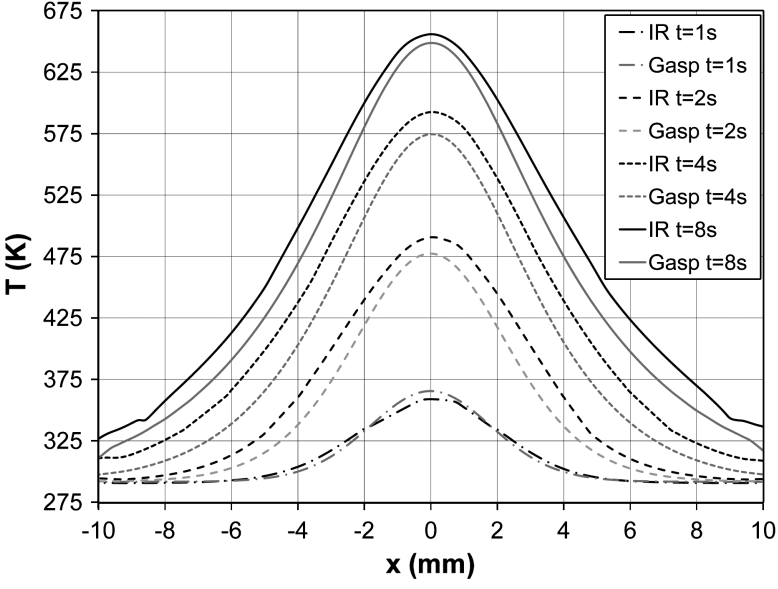

b) Flow-on

Fig. 16 Comparison between infrared measurement and computed backside temperature as a function of time at $P=81 \mathrm{~W}$.

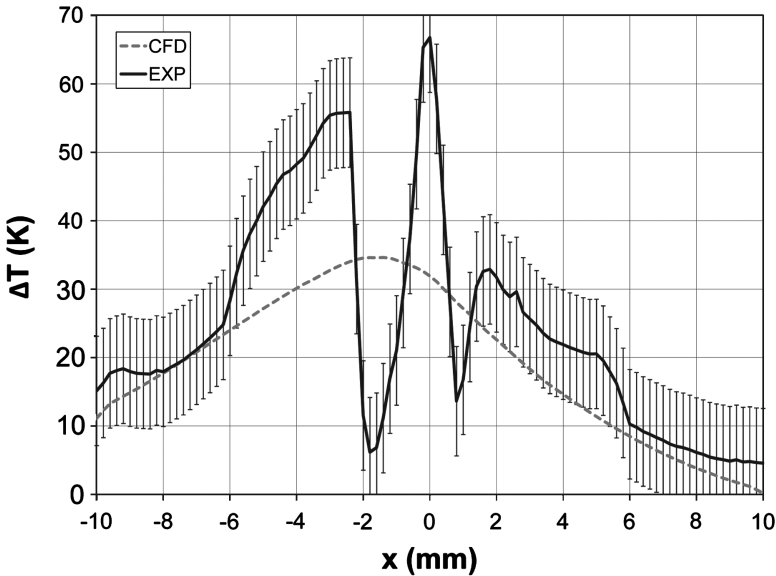

a) Temperature diffenence at the surface for $t=10 \mathrm{~s}$

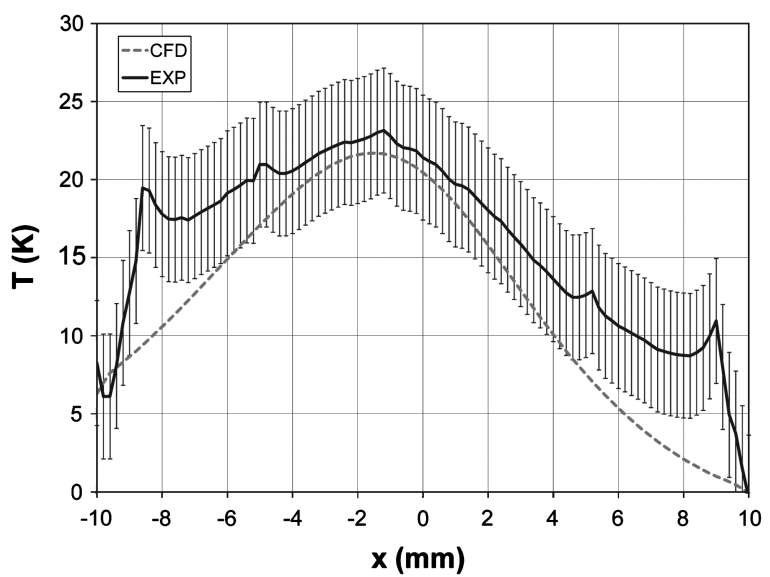

b) Temperature diffenence on the backside for $t=8 \mathrm{~s}$

Fig. 17 Comparison of the temperature difference between CFD and experiment for $P=81 \mathrm{~W}$ (with error bars).

For both the backside and surface, the temperature is underpredicted by the computation, with the exception of $t=0.5 \mathrm{~s}$ for the flow-off case. However, this difference is contained in the uncertainty margin when taking into account the bias error. Here, experimental results indicate that the laser power is probably greater than that reported by the manufacturer. The temperature difference of $60 \mathrm{~K}$ observed between the computation and the experiment at $t=10 \mathrm{~s}$ corresponds to a difference of only $7 \%$ in the power level. The dependence of the emissivity on the temperature could also explain the discrepancy between the simulation and experiment. However, because the value of emissivity as a function of temperature for the paint is not known, this remains speculation. A rise in emissivity of $10 \%$ would lead to an increase in temperature of $30 \mathrm{~K}$. The difference between the experiment and the computation is less for the flow-on case, because the measured temperature decrease due to the flow is greater than that computed. At $t=0.5 \mathrm{~s}$, significant asymmetry is found in the 


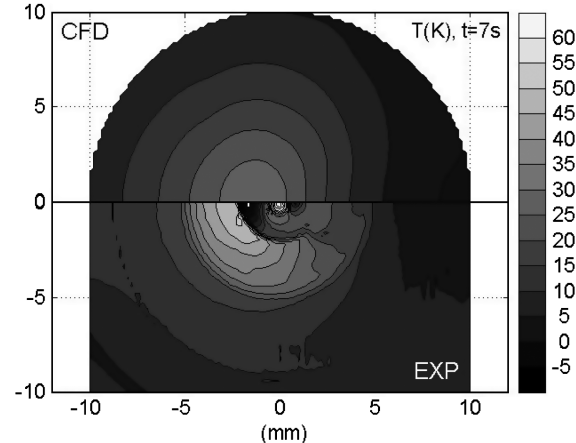

a) Surface

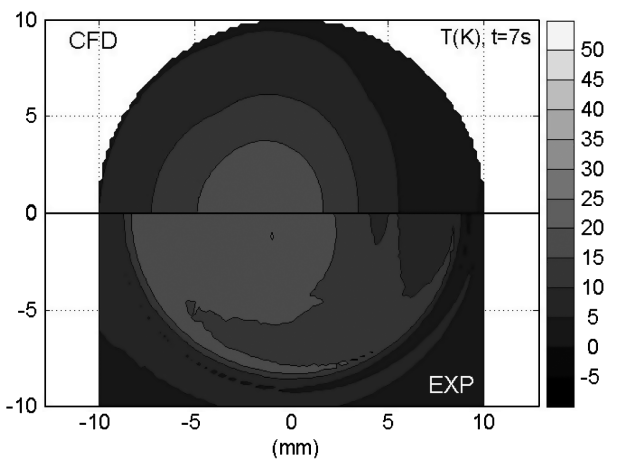

b) Backside

Fig. 18 Comparison of temperature difference between CFD and experiment for $P=81 \mathrm{~W}$ at $t=7 \mathrm{~s}$. Computation on top and experiment on bottom.

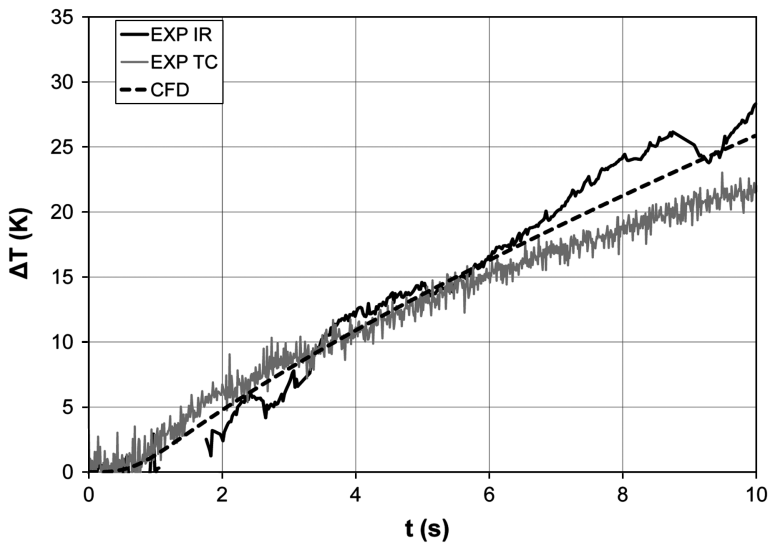

a) Backside temperature difference at $\mathrm{x}=-\mathbf{0 . 1}$ inch

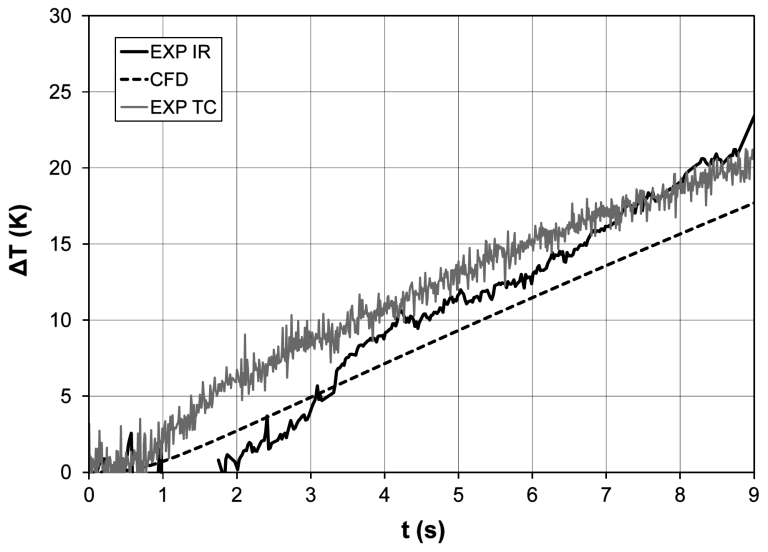

b) Backside temperature at $\mathrm{x}=-\mathbf{0 . 1}$ inch

Fig. 19 Comparison between infrared and thermocouples measurement and computed backside temperature difference as a function of time for $P=81 \mathrm{~W}$.

measured temperature for the flow-on condition, contrary to the computation that appears to be almost perfectly symmetric. With an increase in time, this asymmetry is convected further downstream between $x=4$ and $6 \mathrm{~mm}$.

The temperature difference profiles on the surface at $t=10 \mathrm{~s}$ and on the backside at $t=8 \mathrm{~s}$ are shown in Figs. 17a and $17 \mathrm{~b}$. The temperature is underpredicted at the center. At $\bar{t}=10 \mathrm{~s}$, the measured temperature difference exceeds $60 \mathrm{~K}$, compared with $30 \mathrm{~K}$ for the computation. At $65 \mathrm{~W}$, the underprediction was less, as the measured difference reached $50 \mathrm{~K}$, compared with $31 \mathrm{~K}$ for the computation. Upstream of this intense cooling region, good agreement was found between the computation and the experiment. This situation is clearly visualized in Fig. 18a, in which the computed surface temperature is plotted on the upper half and the measurement is on the lower half. This means that the level of cooling before the perturbation introduced by the strong heating seems to be well modeled. Downstream, away from the center, good agreement is also seen, which leads one to think that the boundary layer relaxes quickly from its heat-induced perturbation. On the backside, good agreement is generally found, but more cooling is measured upstream, as shown in Fig. 18b; this is consistent with the surface prediction.

As shown in Fig. 17a, the measured temperature displays an oscillatory behavior close to the center. The interface between the region of cooling and heating probably is not as sharp as the measurements indicate, because heat diffusion should smooth the gradient out unless a strong flow feature such as a vortex is able to maintain it. For instance, one can imagine strong heat extraction dragging the heat aft such that the downstream wall temperature is increased. The oscillation in the temperature distribution at the center should damp out with diffusion inside the solid. This can be explained to some extent, because a different surface temperature distribution can lead to a similar temperature distribution on the back. Also, we have noticed that for both the 65 and the $81 \mathrm{~W}$ cases, the greatest temperature difference arises near the center over a very small area. Moreover, the area of intense cooling is surrounded by an area of heating, such that the difference in cooling near the center is not as large as it appears.

The disagreement at the center could be due to the incapacity of the turbulence model to respond to the strong distortion of the boundary layer. Specifically, in the turbulence models, only the solenoidal part of the dissipation rate is modeled, and the terms due to fluctuations in viscosity are neglected as is the compressible term. Because there is a large temperature gradient inside the boundary layer, we can expect large viscosity fluctuations, such that the viscosity fluctuation terms in the dissipation rate equation may become important. The same can be said about the density, as a large density gradient exists in the boundary layer. Therefore, the validity of Morkovin's hypothesis [23], which assumes that small density fluctuation is questionable for a flow over a laser-heated wall. The constant-turbulent-Prandtlnumber assumption is also questionable, as it implies a similarity between the velocity and thermal fields. One can compute a variable turbulent Prandtl number by solving two extra transport equations [24,25]: namely, one for the variance of the temperature fluctuation and the other for its dissipation rate. Future work regarding such models could be pursued when trying to improve the prediction of the surface temperature near the beam center.

On the backside, good agreement is also seen between the infrared measurements, thermocouple measurements, and computation, as shown in Figs. 19a and 19b, in which the temperature difference as a function of time is plotted. The similarity between the two independent measurement methods demonstrates the reliability of the measurement systems. 


\section{Conclusions}

Experiments on a laser-heated target subjected to a supersonic turbulent flow have shown that an asymmetry in the temperature distribution increases with laser power. Despite this effect, the maximum decrease in wall temperature near the beam center, due to convective cooling, remains proportional to the laser power. The constant of proportionality corresponds to the ratio of convective cooling to laser heating, which is equal to $7 \%$ for the current experiments. This simple scaling is effective, because the time rate of change of the surface temperature quickly decreases such that a quasi-steady state is achieved. Therefore, excluding short times, the change in convective cooling is small because it directly scales with the temperature increase, which varies slowly over large times. The time scale corresponding to the quasi-steady state is related to the beam waist $w$ and thermal diffusivity of the solid $\alpha_{s}$, such that it is useful to form a dimensionless time $t^{*}=t w^{2} / \alpha_{s}$. The experiments show that the scaling is not very good for a dimensionless time smaller than 3 , which corresponds to $t=1.1 \mathrm{~s}$ for the current experiments. As a consequence of the scaling, the maximum temperature increase with flow for $t / \tau>3$ can be computed from the flow-off temperature when multiplied by $93 \%$, which corresponds to the heat flux reduction near the center. The scaling of the flow-off temperature is not valid downstream, away from the center. Over that region, convective cooling is less, due to the lower wall temperature, such that the effect of the hot plume has a significant effect on the cooling-rate reduction. For a fixed geometry and plate material, the maximum convective cooling is expected to increase linearly with Stanton number and momentum flux $\rho_{e} u_{e}$ at the edge of the boundary layer. However, because conjugate effects are also present, cooling will be affected by the thermal properties of the solid and the beam-waist-to-plate-thickness ratio $w / L$. Conjugate effects should scale with the conjugate Peclet number and $w / L$. Further experiments or simulations would be required to precisely quantify the effect of the flow conditions, geometry, and thermal properties of the solid on the cooling.

Unsteady RANS conjugate heat transfer simulations of the experiments have shown that the heated spot acts as a bump, which deflects the flow upward from the pressure increase. Disturbances propagating along the Mach lines are seen. Such laser-generated disturbances might be used for flow control. Close to the center, the measurements display significantly more cooling than the computations. The scaling seen in the experiments is not exactly reproduced by the computation, as the convective cooling to laser heating at the center reaches $5 \%$ at $65 \mathrm{~W}$, compared with $4 \%$ at $81 \mathrm{~W}$. Downstream, away from the center, good agreement is seen, which indicates that the boundary layer relaxes quickly from its heatinduced perturbation. Disagreement near the center might be due to the incapacity of the turbulence model to respond to the strong distortion of the boundary layer. The development of new turbulence models for strongly heated flow might be needed that do not rely on Morkovin's hypothesis [23] and the assumption of a similarity between the temperature and velocity fields. The experimental methodology developed in this paper could be used for the development and validation of such models.

\section{Acknowledgment}

This work was funded by Arnold Engineering Development Center (AEDC) through the U.S. Air Force Small Business Innovation Research (SBIR) project under contract FA9101-04-C0035 .

\section{References}

[1] Horkovich, J. A., "Directed Energy Weapons: Promise and Reality," AIAA Paper 2006-3753, 2006.

[2] Debieve, J., Dupont, P., Smith, D., and Smits, A., "Supersonic Turbulent Boundary Layer Subjected to Step Changes in Wall Temperature," AIAA Journal, Vol. 35, No. 1, Jan. 1997, pp. 51-57. doi: $10.2514 / 2.86$
[3] Marineau, E. C., Schetz, J. A., and Neel, R. E., "Turbulent NavierStokes Simulations of Heat Transfer with Complex Wall Temperature Variations," Journal of Thermophysics and Heat Transfer, Vol. 21, No. 3, 2007, pp. 525-535. doi: $10.2514 / 1.26007$

[4] Menart, J., Henderson, S., Atzbach, C., Shang, J., Kimmel, R., and Hayes, J., "Study of Surface and Volumetric Heating Effects in a Mach 5 Flow," AIAA Paper 2004-2262, 2004.

[5] Kao, K.-H., and Liou, M.-S., "Application of Chimera/Unstructured Hybrid Grids for Conjugate Heat Transfer," AIAA Journal, Vol. 35, No. 9, 1997, pp. 1472-1478. doi: $10.2514 / 2.270$

[6] Bautista, O., Mendez, F., and Luna, N., "Cooling of a Heat-Generating Strip Immersed in a Laminar Channel Flow," Journal of Thermophysics and Heat Transfer, Vol. 20, No. 3, 2006, pp. 415-421. doi: $10.2514 / 1.14923$

[7] Cole, K., "Conjugate Heat Transfer from a Small Heated Strip," International Journal of Heat and Mass Transfer, Vol. 40, No. 11, 1997, pp. $2709-2719$. doi:10.1016/S0017-9310(96)00232-3

[8] Li, Y., and Ortega, A., "Forced Convection from a Rectangular Heat Source in Uniform Shear Flow: The Conjugate Peclet Number in the Thin Plate Limit," The Sixth Intersociety Conference on Thermal and Thermomechanical Phenomena in Electronic Systems, Inst. of Electrical and Electronics Engineers, Piscataway, NJ, 1998, pp. 284 294.

doi:10.1109/ITHERM.1998.689563

[9] Zuccher, S., and Saric, W., "Infrared Thermography Investigations in Transitional Supersonic Boundary Layers," Experiments in Fluids, Vol. 44, No. 1, 2008, pp. 145-157. doi:10.1007/s00348-007-0384-1

[10] Kare, J. T., "Near-Term Laser Launch Capability: The Heat Exchanger Thruster," AIP Conference Proceedings, Vol. 664, American Inst. of Physics, Melville, NY, May 2003, pp. 442-453. doi: $10.1063 / 1.1582132$

[11] Yan, H., Gaitonde, D., and Shang, J., "The Effect of a Thermal Bump in Supersonic Flow," AIAA Paper 2008-1096, 2008.

[12] Marineau, E., "Computational and Experimental Investigation of Supersonic Convection over a Laser-Heated Target," Ph.D. Thesis, Virginia Polytechnic Inst. and State Univ., Blacksburg, VA, May 2007.

[13] Schetz, J., Boundary Layer Analysis, Prentice-Hall, Upper Saddle River, NJ, 1993.

[14] Ferber, M., "Thermal Shock Testing of Advanced Ceramics-Subtask 9, Final Report," Oak Ridge National Lab., Oak Ridge, TN, 2000.

[15] Ferber, M., and Breder, K., "Thermal Shock Testing of Advanced Ceramics-Subtask 9, Draft," Oak Ridge National Lab., Oak Ridge, TN, 1999.

[16] Dury, M., Theocharous, T., Harrison, N., Fox, N., and Hilton, M., "Common Black Coatings-Reflectance and Ageing Characteristics in the $0.32-14.3 \mu \mathrm{m}$ Wavelength Range," Optics Communications, Vol. 270, No. 2, 2007, pp. 262-272. doi:10.1016/j.optcom.2006.08.038

[17] Nakos, J., "Uncertainty Analysis of Steady State Incident Heat Flux Measurements in Hydrocarbon Fuel Fires," Sandia National Labs. TR SAND2005-7714, Albuquerque, NM, 2005.

[18] "Measure Surface Temperature with a Response Time as Little as 1 Microsecond," Medtherm Corp., Bulletin 500, Huntsville, AL, 1994.

[19] Roe, P. L., "Approximate Riemann Solvers, Parameter Vectors, and Difference Schemes," Journal of Computational Physics, Vol. 43, No. 2, 1981, pp. 357-372.

[20] GASP, Software Package, Ver. 3.0, AeroSoft, Inc., Blacksburg, VA, 1997

[21] Marineau, E. C., Schetz, J. A., and Neel, R. E., "Turbulent NavierStokes Simulations of Heat Transfer with Complex Wall Temperature Variations," AIAA Paper 2006-3087, June 2006.

[22] Roache, P., Verification and Validation in Computational Science and Engineering, Hermosa, Albuquerque, NM, 1998.

[23] Morkovin, M. V., "Effect of Compressibility on Turbulent Flows," Mecanique de la Turbulence, edited by A. Favre, Éditions du Centre National de la Recherche Scientifique, Paris, 1962, pp. 367.

[24] Sommer, T., So, R., and Zhang, H. S., "Near-Wall Variable-PrandtlNumber Turbulence Model for Compressible Flows," AIAA Journal, Vol. 31, No. 1, Jan. 1993, pp. 27-35. doi: $10.2514 / 3.11314$

[25] Brinckman, K., Kenzakowski, D., and Dash, S., "Progress in Practical Scalar Fluctuation Modeling for High-Speed Aeropropulsive Flows," AIAA 43rd Aerospace Sciences Meeting, Reno, NV, AIAA Paper 20050508, Jan. 2005 